\title{
The broad phenotypic spectrum of 17 $\alpha$-hydroxylase/17,20-lyase (CYP17A1) deficiency: a case series
}

\author{
Min Sun 1,2, Jonathan W Mueller 1,2, Lorna C Gilligan 1,2, Angela E Taylor ${ }^{1,2}$, Fozia Shaheen ${ }^{1,2}$, Anna Noczyńska ${ }^{3}$, \\ Guy T'Sjoen ${ }^{4}$, Louise Denvir5, Savitha Shenoy6, Piers Fulton?7, Timothy D Cheetham ${ }^{8}$, Helena Gleeson ${ }^{2,9}$, \\ Mushtaqur Rahman ${ }^{10}$, Nils P Krone ${ }^{11}$, Norman F Taylor ${ }^{12}$, Cedric H L Shackleton ${ }^{1,2,13}$, Wiebke Arlt ID $^{1,2}$ and \\ Jan Idkowiak 1,2,14
}

${ }^{1}$ Institute of Metabolism and Systems Research, College of Medical and Dental Sciences, University of Birmingham, Birmingham, UK, ${ }^{2}$ Centre for Endocrinology, Diabetes and Metabolism, Birmingham Health Partners, University of Birmingham and University Hospitals Birmingham NHS Foundation Trust, Birmingham, UK, ${ }^{3}$ Department of Endocrinology and Diabetology for Children and Adolescents, Wroclaw Medical University, Wroclaw, Poland, ${ }^{4}$ Department of Endocrinology, Ghent University Hospital, Ghent, Belgium, ${ }^{5}$ Department of Paediatric Endocrinology and Diabetes, Queen's Medical Centre, Nottingham, UK, ${ }^{6}$ Children's and Adolescent Services, University Hospitals of Leicester NHS Trust, Leicester, UK, ${ }^{7}$ West Midlands Regional Genetics Service, Birmingham Women's and Children's NHS Foundation Trust, Birmingham, UK, ${ }^{8}$ Newcastle University c/o Department of Paediatric Endocrinology, Royal Victoria Infirmary, Newcastle Upon Tyne, UK, ${ }^{9}$ Department of Endocrinology, University Hospitals Birmingham NHS Foundation Trust, Birmingham, UK, ${ }^{10}$ Department of Endocrinology, Northwick Park Hospital, London Northwest University Healthcare NHS Trust, London, UK, ${ }^{11}$ Academic Unit of Child Health, Department of Oncology \& Metabolism, University of Sheffield, Sheffield, UK, ${ }^{12}$ Department of Clinical Biochemistry, King's College Hospital, London, UK, ${ }^{13}$ Benioff Children's Hospital, University of California San Francisco, Oakland, California, USA, and ${ }^{14}$ Department of Endocrinology and Diabetes, Birmingham Children's Hospital, Birmingham Women's and Children's NHS Foundation Trust, Birmingham, UK

Correspondence should be addressed to J Idkowiak Email j.idkowiak@bham.ac.uk

\begin{abstract}
Context: 17 $\alpha$-Hydroxylase/17,20-Iyase deficiency (17OHD) caused by mutations in the CYP17A1 gene is a rare form of congenital adrenal hyperplasia typically characterised by cortisol deficiency, mineralocorticoid excess and sex steroid deficiency.

Objective: To examine the phenotypic spectrum of $170 \mathrm{HD}$ by clinical and biochemical assessment and corresponding in silico and in vitro functional analysis.

Design: Case series.

Patients and results: We assessed eight patients with 17OHD, including four with extreme 17OHD phenotypes: two siblings presented with failure to thrive in early infancy and two with isolated sex steroid deficiency and normal cortisol reserve. Diagnosis was established by mass spectrometry-based urinary steroid profiling and confirmed by genetic CYP17A1 analysis, revealing homozygous and compound heterozygous sequence variants. We found novel (p.Gly111Val, p.Ala398Glu, p.Ile371Thr) and previously described sequence variants (p.Pro409Leu, p.Arg347His, p.Gly436Arg, p.Phe53/54del, p.Tyr60llefsLys88X). In vitro functional studies employing an overexpression system in HEK293 cells showed that 17,20-lyase activity was invariably decreased while mutant $17 \alpha$-hydroxylase activity retained up to $14 \%$ of WT activity in the two patients with intact cortisol reserve. A ratio of urinary corticosterone over cortisol metabolites reflective of $17 \alpha$-hydroxylase activity correlated well with clinical phenotype severity. Conclusion: Our findings illustrate the broad phenotypic spectrum of 17OHD. Isolated sex steroid deficiency with normal stimulated cortisol has not been reported before. Attenuation of $17 \alpha$-hydroxylase activity is readily detected by urinary steroid profiling and predicts phenotype severity.
\end{abstract}

\section{Significance statement}

Here we report, supported by careful phenotyping, genotyping and functional analysis, a prismatic case series of patients with congenital adrenal hyperplasia due to 17 $\alpha$-hydroxylase (CYP17A1) deficiency (17OHD). These range

https://eje.bioscientifica.com https://doi.org/10.1530/EJE-21-0152 Printed in Great Britain

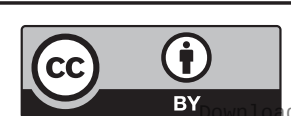

This work is licensed under a Creative Commons Attribution 4.0 International License. 
in severity from the abolition of function, presenting in early infancy, and unusually mild with isolated sex steroid deficiency but normal ACTH-stimulated cortisol in adult patients. These findings will guide improved diagnostic detection of CYP17A1 deficiency.

\section{Introduction}

The classic phenotypic presentation of $17 \alpha$-hydroxylase deficiency (17OHD) comprises cortisol and sex steroid deficiency combined with mineralocorticoid excess (1). Affected genetic males $(46, \mathrm{XY})$ commonly present with female external genitalia at birth, are usually raised as girls and are often not diagnosed until they reach adolescent age when they present with pubertal delay and hypertension, similar to $46, \mathrm{XX}$ females. Patients typically escape the adrenal crisis, despite being profoundly cortisol deficient, due to the affinity of accumulating corticosterone for the glucocorticoid receptor $(1,2)$.

The molecular bases of 17OHD are inactivating mutations in the CYP17A1 gene, coding for an enzyme that is located at a major branch point in human steroidogenesis. CYP17A1 (also P450c17) exhibits two distinct catalytic activities. CYP17A1 $17 \alpha$-hydroxylase activity is crucial for cortisol biosynthesis in the adrenal by converting progesterone and pregnenolone to 17-hydroxyprogesterone (17OHP) and 17-hydroxypregnenolone (17Preg), respectively, the 21-carbon precursors of cortisol. CYP17A1 17,20-lyase activity generates sex steroids in adrenal and gonads by converting $17 \mathrm{OHP}$ to androstenedione and, with about 50-fold higher efficiency (3), 17Preg to DHEA, the principal androgen precursor in humans. CYP17A1 requires electron transfer from NAD phosphate (NADPH). For sufficient reaction efficiency, the flavoprotein $\mathrm{P} 450$ oxidoreductase (POR) is crucial for both catalytic steps (4, 5 ) and the small haemoprotein cytochrome b5 (CYB5A) for efficient 17,20-lyase activity (6).

To date, more than 100 mutations in the CYP17A1 gene have been described (www.hgmd.cf.ac.uk), and the majority are associated with a classic phenotype of combined 17 $\alpha$-hydroxylase/17,20-lyase deficiency. A smaller number of $C Y P 17 A 1$ missense variants are reported to exhibit partial impairment of $17 \alpha$-hydroxylase/17,20lyase activity, where hypertension is mild or absent and external genitalia appear ambiguous in $46, \mathrm{XY}$ individuals $(7,8,9,10,11)$. A small number of individuals affected by $17 \mathrm{OHD}$ present with apparently isolated 17,20-lyase deficiency, with abolished or severely reduced 17,20lyase activity but only mild to moderate impairment of $17 \alpha$-hydroxylase activity $(12,13,14)$. These patients had normal blood pressure and random cortisol levels but reduced sex steroid production. This suggests that variations in CYP17A1 17 $\alpha$-hydroxylase activity determine the width of the phenotypic spectrum of 17OHD.

We have analysed a series of eight patients with 17OHD including four patients with novel phenotypes at opposite ends of the phenotypic spectrum: two young siblings with indications of glucocorticoid deficiency at one end and two patients with isolated sex steroid deficiency and normal stimulated cortisol levels after ACTH stimulation at the other. We characterised corresponding CYP17A1 enzymatic activities in vivo, utilising urinary steroid profiling by gas chromatography-mass spectrometry (GCMS), and in vitro, employing a mammalian overexpression system as well as in silico modelling.

\section{Patients and methods}

Informed consent and assent, if applicable, to participate in this study were obtained from patients and parents, respectively. Patients 1-5 were recruited in the study from the UK Gonadal and Adrenal Inherited Disorders Network (Institute of Child Health/ Great Ormond Street Hospital Research Ethics Committee; REC number 07/Q0508/24). In patients $6-8$, generic consent forms were employed that were designed following guidance published by the Committee on Publication Ethics (COPE) on Journal's Best Practices Ensuring Consent for Medical Case Reports (www.publicationethics.org).

Case 1: The baby $(46, \mathrm{XX})$ was born at 38 weeks gestation by emergency caesarean section due to foetal distress; birth weight was $2530 \mathrm{~g}$ ( -1.8 SDS). Parents were consanguineous. She was well immediately after birth, not requiring neonatal intensive care support and was discharged on day 2 of life. She was fully bottle-fed, drinking $90 \mathrm{~mL}$ term formula every $3-4 \mathrm{~h}$. At 5 weeks, she was assessed for prolonged jaundice and poor weight gain (weight 2640 
$\mathrm{g},-4$ SDS). There was no recorded hypoglycaemia. Her total bilirubin was $194 \mu \mathrm{mol} / \mathrm{L}$ (normal <20) with a conjugated fraction of $41 \mu \mathrm{mol} / \mathrm{L}$. Investigations for prolonged conjugated jaundice, including an ultrasound of her liver, were normal, but random cortisol was $25 \mathrm{nmol} / \mathrm{L}$ and did not increase after stimulation with synacthen (Table 1). Plasma ACTH was highly elevated, suggesting primary adrenal insufficiency. 17OHP was undetectable. Serum potassium and sodium levels were within the normal range. Physical examination showed normal female external genitalia. Findings of urinary steroid profiling by GC-MS (described in results below) were consistent with $17 \alpha$-hydroxylase deficiency, prompting initiation of glucocorticoid replacement. Without any further interventions, the girl developed well and remained on a dose of $10-12 \mathrm{mg} / \mathrm{m}^{2} /$ day hydrocortisone.

Case 2: The younger sister of case $1(46, \mathrm{XX})$ presented to the hospital at 8 weeks of age with failure to thrive. She was born small for gestational age with a birth weight of 2.6 $\mathrm{kg}$ at the $42 \mathrm{nd}$ week of gestation (-2.7 SDS). Pregnancy and birth were unremarkable. Due to the loss of follow-up with her older sister (case 1), peri-natal investigations were not initiated. She had been discharged on day 2 after normal vaginal delivery. Her weight on admission was $3.18 \mathrm{~kg}(-3.8$ SDS). Her clinical examination was unremarkable, with normal female external genitalia. A random cortisol was undetectable, the 17OHP was low, and her electrolytes were within the normal range (Table 1). There was no recorded hypoglycaemia. Her total unconjugated bilirubin was 22 $\mu \mathrm{mol} / \mathrm{L}$ (normal $<20$ ). Given the family history, she was started on a maintenance dose of hydrocortisone $(10 \mathrm{mg} /$ $\mathrm{m}^{2} /$ day) and, without any further intervention, she started to gain weight on demand bottle feeds and remained well on ongoing hydrocortisone supplementation. A urinary steroid profile by GC-MS obtained after the initiation of hydrocortisone substitution showed a similar pattern to that of her sister, consistent with 17OHD but with normal concentrations of cortisol metabolites, as would be expected while treated.

Case 3: The patient $(46, \mathrm{XX})$ was born at term with a weight of $2200 \mathrm{~g}$ (-2.7 SDS) as the third child of nonconsanguineous parents of Polish origin. Pregnancy and birth were uneventful. At the age of 2 years, she was diagnosed with hypertension and treatment with $\beta$-blockers and calcium antagonists was initiated. During childhood, the girl was frequently hospitalised due to electrolyte disturbances. At the age of 15 years, she was admitted to the paediatric endocrine service for further investigation of delayed pubertal development and hypertension. She presented with proportional body growth (height $168 \mathrm{~cm}$ (0.93 SDS), weight $49.2 \mathrm{~kg}(-0.55$ SDS)), lack of pubertal development (Tanner stages PH1, B2) and elevated blood pressure (150/90 $\mathrm{mmHg}, 3.56 / 2.98$ SDS for height), with normal sodium and low-normal potassium (Table 1). Further hormonal investigations revealed low plasma renin activity (PRA, Table 1); ultrasound investigations excluded renal and cardiac causes of hypertension. Baseline cortisol was low and did not increase after ACTH stimulation; 17OHP was normal and ACTH was elevated (Table 1). Androstenedione and oestradiol (E2) were low with elevated gonadotrophins, indicating hypergonadotropic hypogonadism (Table 1). Pelvic ultrasound showed a prepubertal uterus. There were two hypoechogenic cysts (25 and $19 \mathrm{~mm}$ in diameter) within an enlarged right ovary $\left(20.7 \mathrm{~cm}^{3}\right)$; the left ovary contained no cysts and was of normal volume $\left(5.7 \mathrm{~cm}^{3}\right)$.

Treatment with dexamethasone $(0.5 \mathrm{mg} /$ day $)$ was initiated, and after 3 days, the blood pressure normalised to $110 / 70 \mathrm{mmHg}$, which was confirmed on further random blood pressure measurements. Two months later, she suffered from acute abdominal pain and an ultrasound examination showed enlargement of both ovarian cysts. Additional treatment of oestradiol $(1 \mathrm{mg} /$ day) in combination with GnRH super agonist (triptorelin embonate, $3.75 \mathrm{mg} / \mathrm{month}$ ) was initiated. Five days later, abdominal pain resolved, and after the second dose of Diphereline, both ovarian cysts regressed completely.

Case 4: The girl $(46, \mathrm{XX})$ was referred to the adult endocrine service at the age of 17 years with primary amenorrhoea. She had been under regular endocrine surveillance since birth due to congenital hypothyroidism. She had been treated for essential hypertension with a calcium antagonist from the age of 15 years. Her hormonal investigations were suggestive of primary ovarian insufficiency. An initial MRI pelvis suggested absence of cervix and uterus, with a streaky left ovary and no right ovary visualised. Pubertal induction was commenced by the paediatric endocrine services at the age of 16 years. Further hormonal investigations revealed low plasma renin activity with normal electrolytes, low cortisol at baseline and after ACTH stimulation together with low 17OHP (Table 1). She was started on regular hydrocortisone $\left(11.5 \mathrm{mg} / \mathrm{m}^{2} /\right.$ day $)$. She remained on levothyroxine, spironolactone and oestradiol patches for pubertal induction. She has developed withdrawal bleeds 1.5 years after initiation of pubertal induction. A transabdominal ultrasound scan of her pelvis, about 1 year after oestrogen supplementation, confirmed the presence of a uterus with oestrogenised endometrium and the presence of ovaries. 


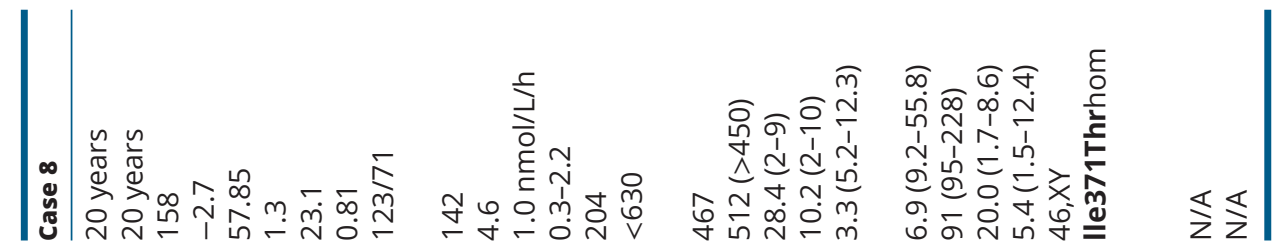

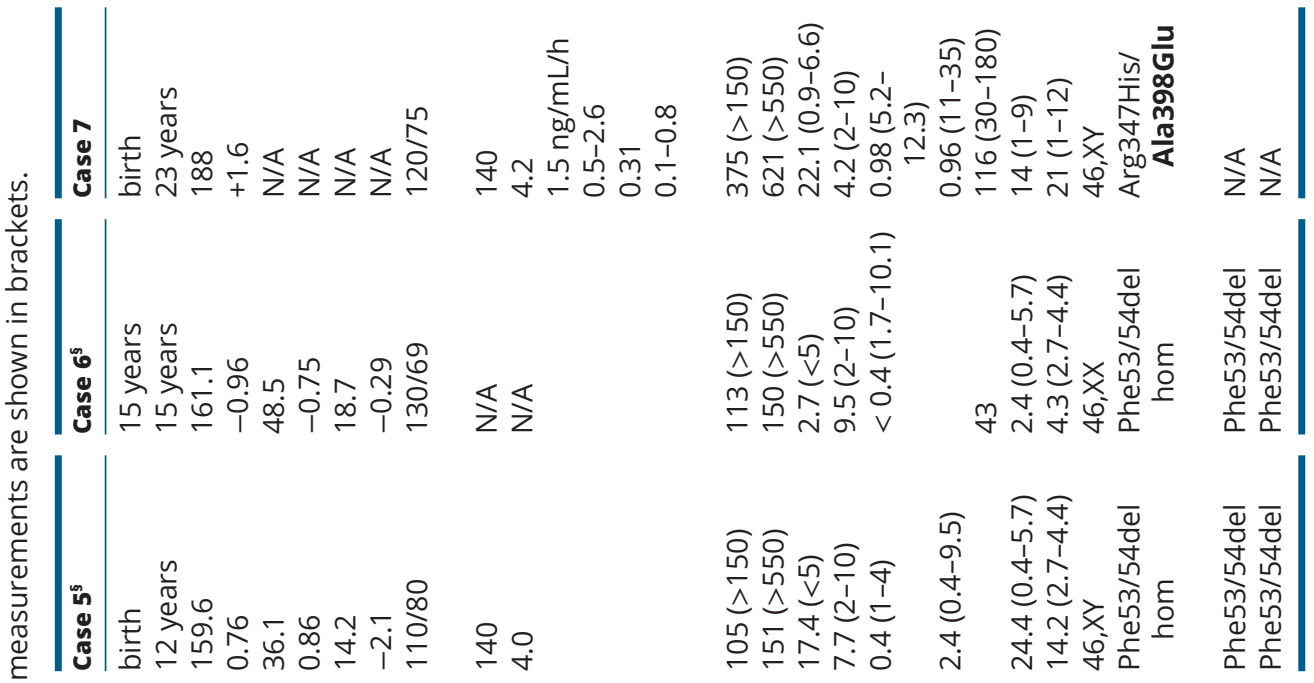

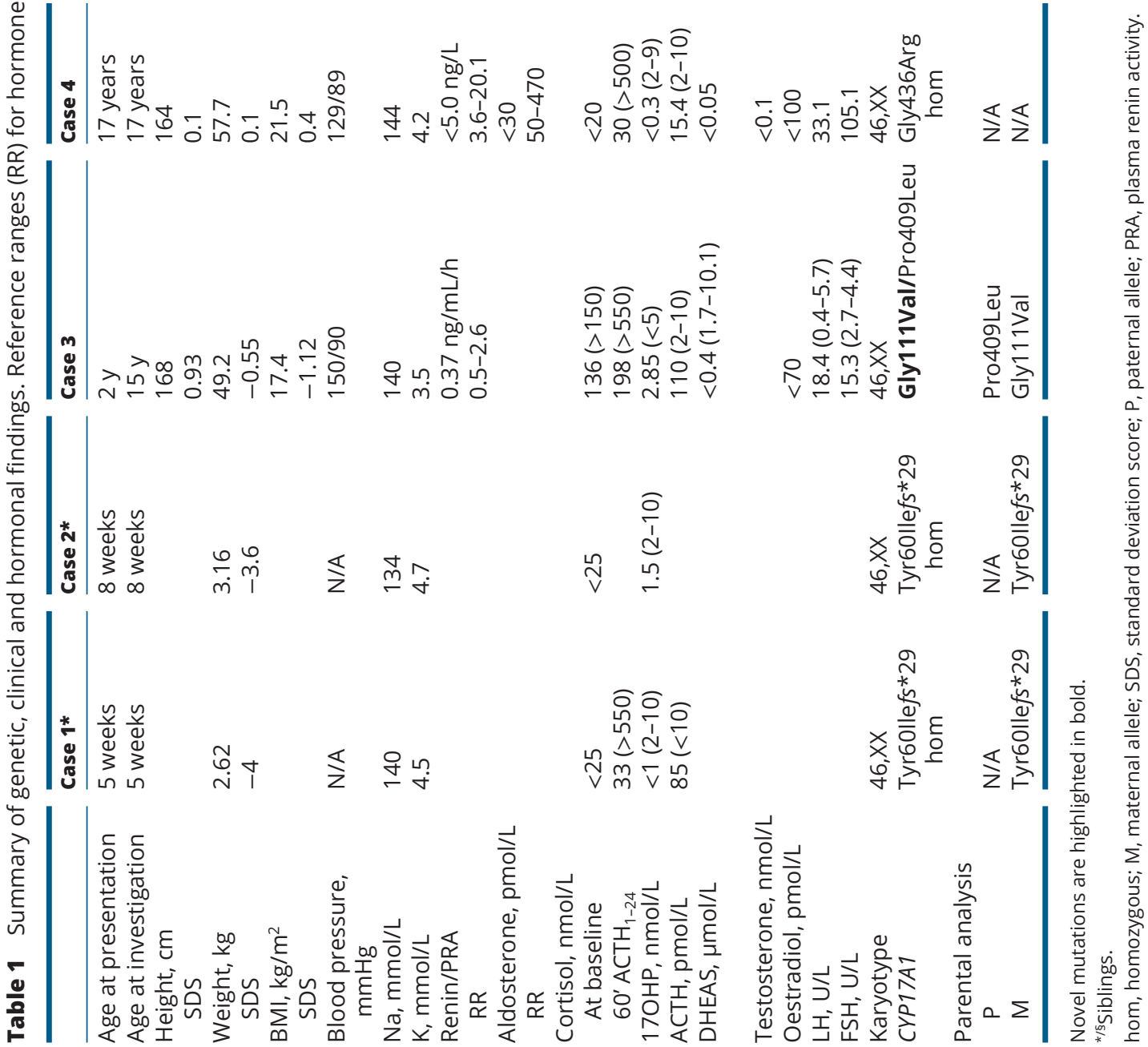


Case 5: The boy $(46, X Y)$ is the fourth child of consanguineous parents of Afghan origin, with no available information on pregnancy and birth. He presented at the age of 12 years with learning difficulties, gynecomastia, micropenis (stretched length $2.5 \mathrm{~cm}$; $<3 \mathrm{rd}$ centile), glandular hypospadias and cryptorchidism and had a history of orchidopexy for a left undescended testis in the age of 5 years. Tanner stages: G1, PH3, testicular volumes were $1 \mathrm{~mL}$ on the right and $4 \mathrm{~mL}$ on the left. His blood pressure was normal, serum potassium and sodium levels were within a normal range (Table 1 ). His serum testosterone was within the lower normal age- and sex-specific range while gonadotrophins were increased, in keeping with compensated hypergonadotropic hypogonadism (Table 1). Baseline cortisol was low and did not increase after i.v. synacthen; $17 \mathrm{OHP}$ at baseline was elevated (Table 1).

Case 6: The older sister $(46, \mathrm{XX})$ of case 5 presented to the endocrine clinic at the age of 15 years because of the family history and delayed pubertal development. Her previous medical history including pregnancy and birth was unremarkable. She had normal breast development (Tanner B4) but there was an absence of menarche and pubic hair growth. Serum cortisol was low at baseline and did not increase sufficiently after i.v. synacthen (Table 1). Serum 17OHP was $2.7 \mathrm{nmol} / \mathrm{L}$ at baseline and increased to $3.5 \mathrm{nmol} / \mathrm{L}$ after synacthen stimulation. Baseline DHEAS was undetectable. Serum oestradiol was prepubertal with gonadotrophins at baseline within the pubertal reference range (Table 1). Blood pressure was normal at several random measurements and within the age- and heightadjusted 50th-90th centiles in a $24 \mathrm{~h}$ blood pressure measurement.

Case 7: The patient $(46, \mathrm{XY})$ was born as the only child of non-consanguineous parents of Caucasian origin in Romania. He presented to the joint endocrine and urology services at the age of 23 years with a wish for phalloplasty and to evaluate his fertility. There are no details available regarding pregnancy, birth and medical history. According to the patient, he was born with a bifid scrotum and an 'absent phallus'. He was raised as a boy and received multiple testosterone injections during childhood and adolescence. He underwent two unspecified genital surgeries, one at 3 months and another at 3 years of age. At the age of 16 years, bilateral gynecomastia was surgically removed. On examination, there was a single perineal opening with no phallus. Both testicles were small; the left testicle was palpable in the inguinal region; the right was found in a bifid scrotum. Blood pressure was normal when measured randomly and over $24 \mathrm{~h}$. Hormonal investigations showed normal cortisol levels at baseline and after synacthen stimulation (Table 1). His 17OHP was elevated (Table 1), a corresponding 17Preg level was normal (7.9 nmol/L; NR $<13.3$ ); the ACTH was within the reference range (Table 1). Sodium, potassium, renin and aldosterone were normal (Table 1). DHEAS and testosterone were both low, with elevated baseline gonadotrophins (Table 1).

Case 8: The patient $(46, \mathrm{XY})$ presented to the adult endocrine service with right-sided gynecomastia at the age of 20 years. He was born in Pakistan to consanguineous parents (first cousins). He reported having two operations to correct hypospadias, completed at the age of 7 years before the family moved to the UK. He has had bilateral mammoplasty during adolescence, but the gynaecomastia was refractory to surgical treatment on the right due to residual breast tissue. On examination, there was no genital ambiguity and his external genitalia appeared male with testicular volumes of 9 (left) and $13 \mathrm{~mL}$ (right). He had appropriate amounts of pubic and axillary hair. His stretched penile length was $9 \mathrm{~cm}$ (below 10th centile). His blood pressure was normal with normal electrolytes and plasma renin activity (Table 1). His 17OHP was mildly elevated and an early morning ACTH was at the upper limit of normal; he had adequate cortisol response after synacthen stimulation (Table 1). Serum testosterone level was slightly below the normal adult male range with elevated LH and normal FSH, indicating hypergonadotropic hypogonadism (Table 1). Imaging studies of his adrenals and testicles did not reveal any pathology; the adrenals were of normal size. He was commenced on testosterone undecanoate $1 \mathrm{~g}$ every 12-14 weeks, following sperm cryopreservation. Following testosterone replacement, he experienced an increase in muscle bulk, beard growth, penile growth and disappearance of palpable breast tissue.

\section{Urinary steroid profiling by gas chromatography- mass spectrometry (GC-MS)}

Analysis of urinary steroid metabolite excretion was performed as described previously by a quantitative GC-MS selected ion-monitoring (SIM) method (15). In brief, steroids were enzymatically released from conjugation and, after extraction, chemically derivatised before GC-MS SIM analysis. Steroids quantified included corticosterone metabolites (tetrahydrocorticosterone

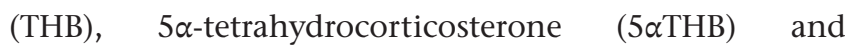
tetrahydro-11-dehydrocorticosterone (THA)), the 17-hydroxyprogesterone metabolites 17-hydroxypregnanolone (17HP) and pregnanetriol (PT), the pregnenolone metabolite pregnenediol (5PD), the 
17-pregnenolone metabolite 5-pregnenetriol (5PT), cortisol metabolites (tetrahydrocortisol (THF), $5 \alpha$-tetrahydrocortisol (5 $\alpha \mathrm{THF}), \quad$ tetrahydrocortisone (THE), cortolones and cortols and androgen metabolites (androsterone (An) and etiocholanolone (Et), DHEA (DHEA)). Additional steroids that are important in the newborn period were quantified in Case 1: 16 $\alpha$-hydroxy-dehydroepiandosterone (16 $\alpha$-OH-DHA), 16 $\alpha$-hydroxypregnenolone 5 $\alpha$ THA, $5 \alpha$ THF and $6 \alpha$-hydroxy tetrahydro-11-dehydrocorticosterone (6a-OH-THA).

Following the quantification of steroid metabolites, we calculated substrate metabolite to product metabolite ratios to determine the approximate in vivo net activities of CYP17A1: corticosterone over cortisol metabolites (17 $\alpha$-hydroxylase; (THA+THB+5 $\alpha \mathrm{THB}) /$ $(\mathrm{THF}+5 \alpha \mathrm{THF}+\mathrm{THE}))$, and 17-hydroxyprogesterone over androgen metabolites (17,20-lyase; (17HP+PT)/(An+Et) and 5PT/DHEA). The diagnostic ratios were compared to data obtained from a healthy reference cohort (males and females, aged $10-30$ years, $n=28$ ).

\section{Sequencing and analysis of sequence variants}

We carried out sequencing analysis for cases 1, 2, 3, 4, 5 and 6 as described previously (16). CYP17A1 gene analysis in genomic DNA from cases 7 and 8 was performed by genetic service laboratories accredited by the National Health Service, England. Sequencing analysis was performed using Lasergene ${ }^{\circ}$ software (DNASTAR Inc., Madison, USA), and mutation numbering was carried out referring to the CYP17A1 NCBI reference sequences Chr10(GRCh38) (genomic), NM_000201.4 (cDNA) and NP_000093 (protein). Allele frequencies and REVEL (Rare Exome Variant Ensemble Learner) scores, an ensemble method for the prediction of missense variants (17), were obtained with the gnomAD 3.1.1 software.

\section{In silico analysis}

The crystal structure of human CYP17A1 (http://www. rcsb.org/pdb, PDB code 3RUK) was used to analyse the impact of non-synonymous sequence variants on the three-dimensional structure of the CYP17A1 enzyme using the Molsoft ICM Browser Pro Software (Molsoft L.L.C, La Jolla, CA, USA).

\section{In vitro functional studies}

The mammalian expression vector pcDNA6-V5-6xHis(B) (Invitrogen) was used for in vitro functional assays in
HEK293 cells. Plasmids containing either the WT human CYP17A1 or mutant sequences were synthesised by GenScript Biotech (Amsterdam, The Netherlands). The stop codon in the ORF was removed to add a C-terminal V5-tag. All constructs were confirmed by Sanger sequencing.

Transfection was carried out as described previously (6). A ratio of $2 \mu \mathrm{g}$ of plasmid DNA to $6 \mu \mathrm{L}$ of FuGENE ${ }^{\oplus} \mathrm{HD}$ transfection reagent per six-well was employed. Fortyeight hours after transfection, cells were incubated at $37^{\circ} \mathrm{C}$ with $1 \mathrm{~mL}$ full MEM supplemented with $1000 \mathrm{nmol} / \mathrm{L}$ progesterone for $17 \alpha$-hydroxylase assays or $500 \mathrm{nmol} / \mathrm{L}$ 17Preg for 17,20-lyase assays. Substrate concentrations were chosen as they are near the Km of the WT enzyme. Optimal incubation times for obtaining linear conversion rates were assessed for WT and mutants in preceding pilot experiments and were $30 \mathrm{~min}$ for $17 \alpha$-hydroxylase and 60 min for 17,20-lyase.

After incubation, $20 \mathrm{ng}$ of each deuterated progesterone, 17OHP, 17Preg, DHEA, and androstenedione (all Cambridge Isotope Laboratories Inc., Andover, MA) was added to the medium as an internal standard to normalise for extraction efficiency. Steroids were extracted from $500 \mu \mathrm{L}$ of spiked medium with $1.5 \mathrm{~mL}$ methyl tertbutyl ether (MTBE, Sigma-Aldrich) as described previously (18). A Waters Xevo mass spectrometer with ACQUITY ultra performance liquid chromatography ${ }^{\mathrm{TM}}$ (UPLC) system was used fitted with an HSS T3, $1.8 \mu \mathrm{M}, 1.2 \times 50 \mathrm{~mm}$ column (Waters Corporation, Milford, MA). Each steroid was quantified by comparison to a calibration series with respect to their internal standard. Quantitation was completed using TargetLynx 4.1 software (MassLynx 4.1, Waters). Conversion rates of CYP17A1 activities were determined as micrograms per milligram of total protein per minute and expressed as a percentage of substrate conversion defining WT activity as $100 \%$. Data analysis was facilitated and graphically illustrated using the GraphPad Prism software version 5.0 (GraphPad Inc. ).

\section{Results}

\section{Sequencing analysis}

The identified CYP17A1 sequence variants are summarised in Table 1 (protein level) and Supplementary Table 1 (see section on supplementary materials given at the end of this article) (full HGVS nomenclature). Parental DNA for segregational analysis was available for cases 1-3, 5 and 6 . Of the eight sequence variants identified, three have not been reported before (p.Gly111Val, p.Ala398G, p.Ile371Thr). Three sequence variants (p.Pro409Leu, 
Gly436Arg and p.Tyr60IlefsLys88X) have been described in clinical case reports but without activity assays $(19,20)$, while reports of two (p.Phe53/54del, p.Arg347His) have included activity assays (10, 14). All sequence variants have low allele frequencies, and pathogenicity prediction of the missense variants revealed REVEL scores of $>0.5$, suggesting that they are likely pathogenic (see Supplementary Table 1).

\section{Urinary steroid profiling}

All patients had received a diagnosis by urinary steroid profiling in various laboratories. Stored samples were reanalysed for this study, with the exception of neonatal cases 1 and 2 and case 4, who had already been started on hydrocortisone replacement (Fig. 1). All showed a typical pattern, with elevated metabolites of steroids in the mineralocorticoid pathway, elevated progesterone and pregnenolone metabolites (5-PT, PT, and 5-PD), but low androgen metabolites (An and $\mathrm{Et}$ ) and borderline to very low metabolites of cortisol (THF, $5 \alpha$-THF, cortolones and cortols). We employed substrate: product ratios reflecting the in vivo catalytic activities for CYP17A1 17 $\alpha$-hydroxylase and 17,20lyase activities. The ratio reflecting $17 \alpha$-hydroxylase activity ((THA+THB+5aTHB)/(THE+THF+5aTHF)) was most severely impaired in case 3 , presenting with a classical phenotype (185.7; NR 0.07-0.18), moderately elevated in cases 5 and 6 , presenting with a moderate phenotype (6.8 and 12.3 ,
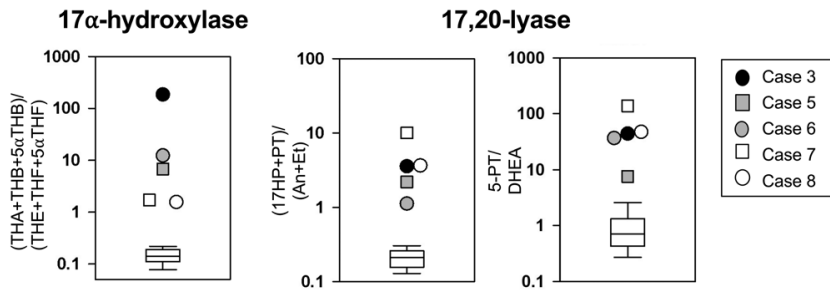

\section{Figure 1}

In vivo assessment of CYP17A1 17 $\alpha$-hydroxylase and 17,20lyase activities as indicated by urinary steroid metabolite analysis. Individual diagnostic steroid metabolite ratios in the five $170 H D$ cases analysed are represented by the symbols as indicated in the legend. Phenotypes with a mild phenotype (cases 7 and 8) are represented as white, intermediate phenotypes (cases 5 and 6) as grey and one case with a severe phenotype (case 3 ) as black symbols. White box plots represent the interquartile ranges of the reference cohort (healthy males and females, $10-30$ years; $n=28$ ), whiskers represent the 5th and 95th percentiles, respectively. For steroid abbreviations please see methods. respectively) and mildly elevated in cases 7 and 8, presenting with a mild phenotype (1.7 and 1.62, respectively) (Fig. 1). We have employed two ratios reflecting CYP17A1 17,20lyase activities: $((17 \mathrm{HP}+\mathrm{PT}) /(\mathrm{An}+\mathrm{Et}))$ in the $\Delta 4$ and $5 \mathrm{PT} /$ DHEA in the $\Delta 5$ pathway. Both ratios were elevated above the reference range for all cases $((17 \mathrm{HP}+\mathrm{PT}) /(\mathrm{An}+\mathrm{Et}): 1.15-$ 10.1 (NR 0.09-0.31); 5PT/DHEA: 7.6-138.3 (NR 0.2-3.7); Fig. 1). GC-MS chromatograms for case 1 (Supplementary Fig 1) showed changes consistent with our previous findings for severely affected newborns (21). These comprise increase of THA + 5 $\alpha$ THA/THE $+5 \alpha$ THE: 37.2 (normal: 0.25 ) and a very high ratio of $16 \alpha$-hydroxypregnenolone /16 $\alpha$-OH-DHEA: $>200$ (normal about unity).

\section{In silico analysis of non-synonymous sequence variants}

Gly111Val (case 3, classic phenotype)

Glycine 111 is in close proximity to extended helix between threonine 294 and threonine 295, down to a distance of $3.9 \AA$ between Gly111 and Thr294. This Thr294 side chain is shown in ball representation and grey in Fig. 2B. Introducing the bulkier valine instead of Gly111 would result in a steric clash, impairing protein function.

\section{Pro409Leu and Gly436Arg (cases 3 and 4, classic phenotypes)}

Proline 409 and Glycine 436 are both located in an extended loop structure ranging from asparagine 402 to Glycine 444 (this loop is highlighted in green in Fig. 2C and F). Glycine 436 is a highly conserved residue, embedded in an extended loop structure in close contact with the haeme co-factor. Gly436 is needed for a hairpin loop beginning at Pro434. Exchanging glycine 436 even with the much bigger arginine will certainly disturb folding and interfere with co-factor binding due to steric clashes. The loop region around Pro409 is rather stable according to structural thermal factors. Most likely, it is the unique properties of the amino acid proline that are required to initiate a tight loop including Asp410 and to stiffen the peptide backbone. Even exchanging proline with the similarly sized hydrophobic amino acid leucine will most likely destabilise the protein fold.

\section{Ala398Glu (case 7, mild phenotype)}

Alanine 398 sits in a relatively polar pocket (Fig. 2D). One of the polar amino acids is glutamine 57, which forms a hydrogen bond with the glycine 77 main chain oxygen. 

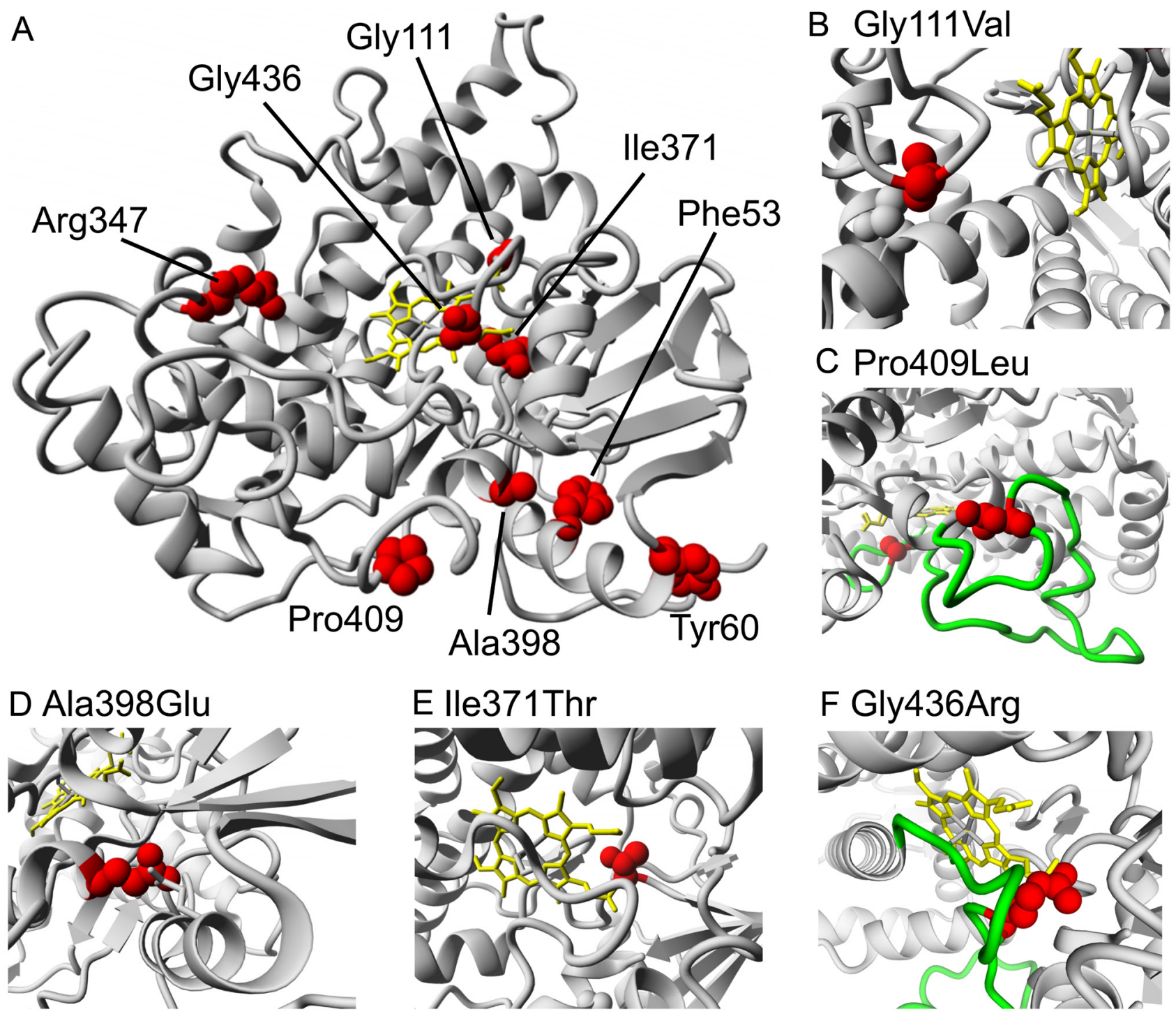

Figure 2

Three-dimensional model of CYP17A1 (RCSB protein databank identifier: 3RUK). Panel A provides an overview of the CYP17A1 model and sequence variants (as WT residues) identified in this case series. Panel B, C, D, E and F magnify the mutated amino acid residues to illustrate the impact of the mutations within their immediate environment.

Introducing glutamate instead of alanine 398 may break this hydrogen bond but could offer opportunities for compensating interactions with glutamine 57 or asparagine 395 as the potential binding partners. Hence, this exchange is expected only to mildly influence protein function.

\section{Ile371Thr (case 8, mild phenotype)}

Isoleucine 371 is in close proximity to arginine $96(3.4 \AA)$ and in spatial proximity to the haem co-factor (Fig. 2E).
Threonine is smaller than isoleucine but fits well into the overall shape of isoleucine. An isoleucine to threonine exchange at this buried site is expected not to cause any steric clash and would impair protein function only mildly.

We have used the structural model for a CYP17A1-b5POR complex (22) to test whether any of the mutations from this study could interfere with redox partner binding. According to this model, none of the mutations described here is likely to interfere with b5 and/or POR binding. 


\section{In vitro functional analysis}

All mutants assessed for the residual enzymatic activity of both catalytic reactions of CYP17A1 showed greatly reduced or absent activity in our cell-based in vitro system (Fig. 3). Four mutants (p.Ile371Thr, p.Arg347His, p.Ala398Glu and p.Phe53/54del) retain some residual activity on $17 \alpha$-hydroxylase function with about $10-15 \%$ of WT activity (Fig. 3A). Amongst these, the p.Ile371His and the p.Arg347Arg mutations, found in the cases 7 and 8 with a mild phenotype, have the highest residual activity $(14.0 \pm 1.2 \%$ and $13.3 \% \pm 2.6 \%$, respectively $)$ with p.Ala398Glu and p.Phe53/54del retaining $5.8 \pm 1.3 \%$ and $5.9 \pm 1.4 \%$, respectively, of WT enzyme activity. In p.Gly111Val, p.Pro409Leu, p.Gly436Arg and Tyr60IlefsLys88X, found in cases with severe phenotypes, $17 \alpha$-hydroxylase activity was abolished in our assay system (Fig. 3A). 17,20-lyase activity was greatly reduced or abolished for all mutant CYP17A1 proteins studied (Fig. 3B). The p.Ile371Thr mutation, found in case 8 with a mild phenotype, retained the highest degree of residual activity (3.6 $\pm 0.9 \%)$. The p.Ala398Glu, p.Phe53/54del and p.Pro409Leu retained residual enzyme activity at $1.5 \%$ of wild-type. The residual 17,20-lyase activity is abolished for the p.Arg347His, the p. Gly111Val, the Gly436Arg and the frameshift mutations (Fig. 3B).

\section{Discussion}

Our case series refines the phenotypic spectrum of 17OHD, describing eight patients with inactivating mutations in the CYP17A1 gene and assessing their functional impact in vivo by urinary steroid profiling analysis and in vitro by functional studies. This showed that residual CYP17A1 $17 \alpha$-hydroxylase activity is associated with the severity of this condition. Four of our cases present with extreme phenotypes: failure to thrive and conjugated hyperbilirubinemia in our siblings (cases 1 and 2) prompted investigations into adrenal insufficiency caused by a severe homozygous frameshift mutation, and, at the other end of the scale, cases 7 and 8 exhibited a normal cortisol increase in response to ACTH.

The majority of patients with combined $17 \alpha$-hydroxylase/17,20-lyase deficiency due to severely inactivating CYP17A1 mutations first present with a lack of pubertal development and low renin hypertension. They may be found to be $46, \mathrm{XX}$, as in cases 3 and 4 , or $46, \mathrm{XY}$. Patients with milder mutations who are $46, \mathrm{XX}$ may present
A $17 \alpha$-hydroxylase

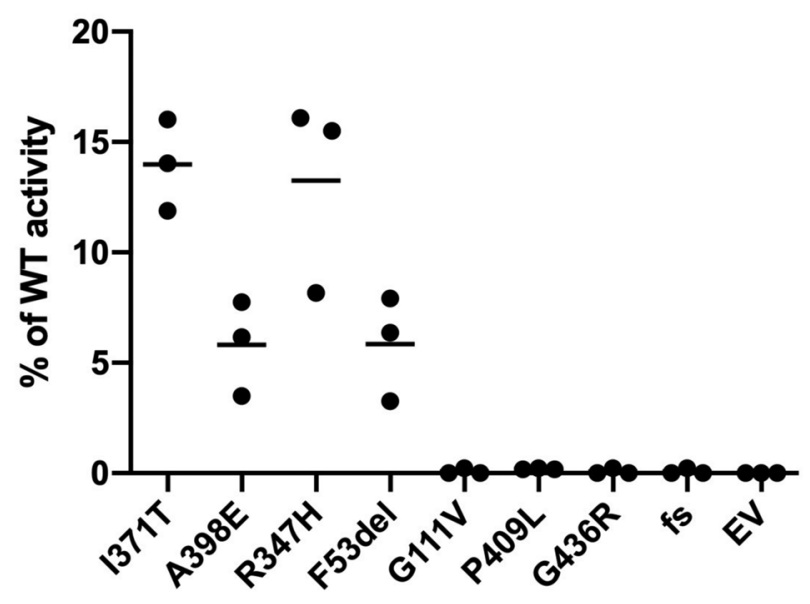

B 17,20-lyase

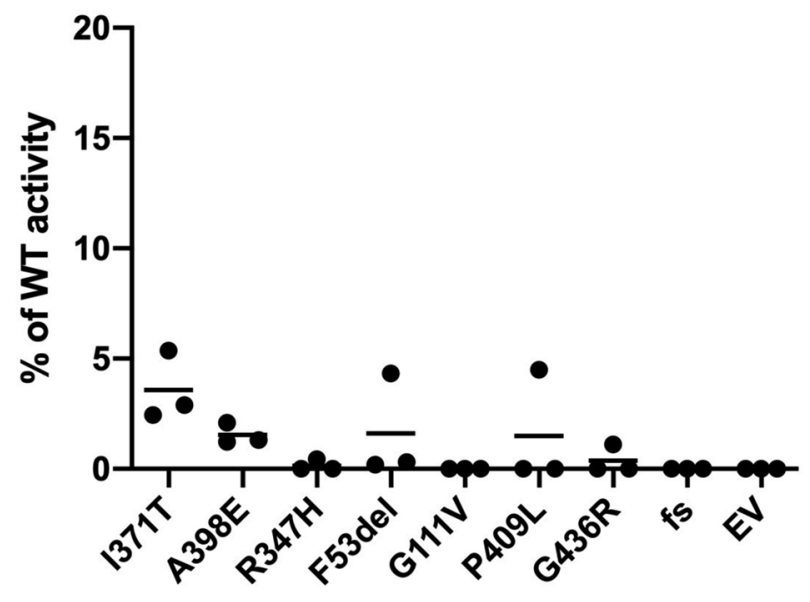

Figure 3

Residual mutant enzyme activity values are shown for the two catalytic activities of CYP17A1. Panel A for the conversion of progesterone (Prog) to 17-hydroxyprogesterone (170HP), reflecting $17 \alpha$-hydroxylase activity; panel $B$ reflects the 17,20-lyase activity as assessed by the conversion of 17-hydroxypregnenolone (17Preg) to DHEA. Residual enzyme activity is expressed at the percentage of WT activity, which is defined as $100 \%$. Substrate conversion rate for WT protein was $14.4 \pm 0.4 \mathrm{nmol} / \mathrm{mg}$ protein/min for Prog and $5.8 \pm 1.5$ $\mathrm{nmol} / \mathrm{mg}$ protein/min for 17Preg. All experiments were performed in triplicate in three independent experiments, and each data point represents the median of each triplicate.

with less overt pubertal delay and be normotensive, as in case 6 , while $46, \mathrm{XY}$ individuals may present at birth with genital ambiguity, although diagnosis may be significantly delayed, as in cases 5, 7 and 8 . 
Cortisol deficiency in 17OHD is appropriately compensated by activation of the HPA axis to maintain the required level of alternative glucocorticoid, primarily corticosterone, an intermediate in the mineralocorticoid pathway $(1,23,24)$. Cases 1 and 2 had increased corticosterone metabolite excretion in the neonatal urinary steroid profile, but failure to thrive, and also conjugated hyperbilirubinaemia in case 1, which may reflect clinical signs of early onset glucocorticoid deficiency. A recent case report of a 46,XX girl harbouring the same frameshift mutation in homozygosity as cases 1 and 2 presented in adolescence with primary amenorrhoea, but details of her neonatal course were not provided (20). Similarly, three Turkish siblings with large-range deletions in CYP17A1 abolishing enzymatic activity presented with severe hypertension and primary amenorrhoea, but early onset adrenal insufficiency has not been reported by the authors (25). While contributing factors such as stress, infections or sepsis are reported to trigger adrenal crisis in 17OHD, as exemplified in the very first reported case by Biglieri (26), these had been excluded at the time of presentation in our siblings. Both siblings recovered after initiation of physiological doses of hydrocortisone alone, and other causes of failure to thrive and conjugated jaundice were excluded. Importantly, the findings from the urinary steroid profiling analysis were instrumental in excluding other causes of primary adrenal insufficiency (i.e. mutations in NR5A1, StAR, CYP11A1 or HSD3B2). Multi-steroid profiling by LC-MS/MS has also been shown to be highly effective in detecting CYP17A1 deficiency early (27) but was not available. Given the consanguineous background of our siblings, concomitant mutations in other genes causing adrenal insufficiency might have contributed to the phenotype, a rare possibility that we were not able to address. However, it does appear that phenotypic severity varies even in severely predicted CYP17A1 genotypes, as exemplified by a report of an adult prepubertal woman with an early truncation of the CYP17A1 protein (homozygous p.Y27X) who did not even develop hypertension (28). Different phenotypes with the same genotype or even family background are recognised in $\mathrm{CAH}$, and genetic variation in other genes or co-factors of steroidogenesis may contribute to this phenotypic variability (29). 'However, on balance, we are not able to conclusively prove that glucocorticoid deficiency due to 17OHD has caused the symptoms observed in these siblings since electrolyte abnormalities and significant hypotension/shock were absent. The clinical signs were subtle and relatively non-specific, nevertheless prompted the clinician to investigate for adrenal pathology'.
Activation of the HPA axis also drives increased production of the corticosterone precursor, 11-deoxycorticosterone (DOC), a weak mineralocorticoid; 18-hydroxylated products of both steroids also have mineralocorticoid activity. This tends to suppress the renin-angiotensin-aldosterone system via blood pressure increase and hypokalaemia. In severe 17OHD, this is insufficient to compensate for the mineralocorticoid excess, resulting in hypertension and hypokalaemia, as in the classic presentations of patients 3 and 4 . Milder deficiencies can be compensated, as seen in patients 5-8. Notwithstanding the above, about $10 \%$ of patients with severe 17OHD are normokalaemic and normotensive (30). This also suggests that there is considerable phenotypic variability, with perhaps both additional compensatory mechanisms and forms of compensatory failure.

Cases 7 and 8 presented with the clinical and biochemical features of so-called 'isolated 17,20-lyase deficiency'. This is a rare condition and only around 30 cases have been described in the literature $(6,12,13,14,31$, $32)$. Initial reports provided only clinical and hormonal characterisation without identifying a distinct genetic abnormality in six families with 46,XY DSD and two families with $46, \mathrm{XX}$ patients that presented with lack of pubertal development (reviewed and discussed in $(1,6)$ ). So far, 17 cases of 'isolated' 17,20-lyase deficiency with a complete clinical, hormonal, genetic and functional work-up have been reported. Underlying causes were four distinct missense mutations in the CYP17A1 gene (p.Arg347His; p.Arg347Cys p.Arg358Gln; p.Glu305Gly) $(12,13,14)$ and one POR missense mutation (p.Gly539Arg) (31). A total of six patients with CYB5A mutations have been reported so far $(32,33)$ and these exhibit the purest form of isolated 17,20-lyase deficiency. CYB5A exclusively supports CYP17A1 17,20-lyase activity, as shown by comprehensive clinical and biochemical phenotyping as well as in vitro functional analysis in an affected kindred with the p.His44Lys mutation (6).

Case 7 carried the known missense mutation p.Arg347His on one allele (14) and a novel missense mutation p.Ala398Glu on the other allele. According to our in vitro functional analysis, the p.Ala398Glu has only $5.8 \%$ residual activity compared to p.Arg347His, which retains $13.3 \%$ of WT activity. The finding that our patient has normal stimulated cortisol levels is due to the effect of the milder p.Arg347His mutation. Case 8 carries the novel p.Ile371Thr mutation in homozygosity and also presented with some degree of sex steroid deficiency/primary hypogonadism and normal cortisol levels after ACTH stimulation. It is remarkable that his serum testosterone 
concentration was within the lower male reference range despite a severe degree of genital ambiguity at birth. This may indicate that testicular testosterone production during embryonic development is more severely affected compared to puberty, which is surprising since accumulating $17 \mathrm{OHP}$ due to deficient 17,20-lyase in the foetal testis should feed into the alternative pathway towards dihydrotestosterone generation $(4,34)$. Further studies on p.Ile371Thr could explore the possibility that this CYP17A1 variant has reduced affinity for 5-pdiol, the substrate of 17,20-lyase in the alternative pathway. Genital ambiguity in $46, \mathrm{XY}$ has a relatively high population prevalence and a molecular basis is not established in most cases (35), so a multi-factorial cause in our patient is possible. The data from our in vitro functional analysis concur with the clinical findings: the $17 \alpha$-hydroxylase activity was $14 \%$, a similar level to the p.Arg347His mutation, but it also retained some residual 17,20-lyase activity.

At the time of assessment, 46,XY patients 7 and 8 had high serum levels of $17 \mathrm{OHP}$, as did case 5 , who had a much higher level than his $46, \mathrm{XX}$ sibling, case 6 . This likely reflects predominantly enhanced testicular $17 \mathrm{OHP}$ production under LH hyperstimulation. This is supported by the urine steroid data, which showed increases above normal of the 17OHP metabolites 17HP and PT but not of 11-oxopregnanetriol, the major metabolite of 21-deoxycortisol and a marker of adrenal 17OHP excess. This indicates a predominantly testicular origin for the 17OHP. Prepubertal XY patients with 17OHD have been reported to show increased 17OHP after hCG stimulation but not after synacthen.

To our knowledge, cases 7 and 8 have the mildest ever reported phenotype for disease-causing mutations in CYP17A1 and are probably closest to what was previously described as 'isolated' 17,20-lyase deficiency in the context of 17OHD. A unifying characteristic of all individuals with 'isolated' 17,20-lyase deficiency is sex steroid deficiency, with hormonal measurements confirming diminished adrenal and gonadal androgen synthesis. All previously reported patients with an apparent isolated 17,20-lyase deficiency with underlying CYP17A1 or POR mutations have shown impairment of cortisol production, with insufficient responses to ACTH stimulation (6). In contrast, cases 7 and 8 are the first reported 17OHD patients who have normal ACTH-stimulated cortisol levels. Nevertheless, urinary steroid analysis demonstrated impairment of $17 \alpha$-hydroxylase activity. Compared to the ratios we have obtained from patients with classical 17OHD or partial combined 17 $\alpha$-hydroxylase/17,20-lyase deficiency (cases 5 and 6), their corticosterone/cortisol metabolite ratios are lower, suggesting higher residual in vivo CYP17A1 $17 \alpha$-hydroxylase activity in milder cases. Thus, a precursor/ product ratio of corticosterone over cortisol metabolites reflecting in vivo $17 \alpha$-hydroxylase activity correlates well with the severity of the disease and may help to predict the phenotype (Fig. 1). This finding is in accord with two previous studies employing GC-MS steroid metabolome analysis in 17OHD subjects with severe (36) and milder (37) disease manifestation. Interestingly, the diagnostic ratios reflecting CYP17A1 17,20-lyase activity in our patients are higher in milder cases (cases 7 and 8) suggesting severe impairment (Fig. 1). Severe 17 $\alpha$-hydroxylase attenuation in more severe disease results in the reduction of 17-hydroxylated metabolites that build the numerator for both ratios. Hence, neither 17,20-lyase ratios reflect the in vivo residual activity in CYP17A1 due to concomitant $17 \alpha$-hydroxylase inhibition.

In our functional analysis, we have not performed enzyme kinetic studies in WT and mutant protein to determine Vmax and $\mathrm{Km}$, thus the reported residual activity at a fixed concentration, around the $\mathrm{Km}$ of the substrates of the WT protein, is a combination of both effects. This is a limitation of our work, however, enables the determination of the disease-causing effects of the variants and characterises their differential impact on $17 \alpha$-hydroxylase and 17,20-lyase activities.

In summary, our case series illustrates the broad phenotypic variability of CYP17A1 deficiency and, importantly, expands both ends of this spectrum. These range from early presentation to isolated sex steroid deficiency with normal stimulated cortisol production, respectively. Urinary steroid profiling by mass spectrometry represents a powerful diagnostic tool not only in establishing the diagnosis but also in predicting the severity of the phenotype (38).

\section{Supplementary materials}

This is linked to the online version of the paper at https://doi.org/10.1530/ EJE-21-0152.

\section{Declaration of interest}

The authors declare that there is no conflict of interest that could be perceived as prejudicing the impartiality of this study.

\section{Funding}

This work was supported by the Medical Research Council UK (Research Fellowship G1001964, to J I, and Program Grant 0900567, to W A), the European Society for Paediatric Endocrinology (Research Fellowship, to J I), the Academy of Medical Sciences (Starter Grant for Clinical Lecturers SGL020/1013, to I I), and the National Institute of Health Research 
(Academic Clinical Lectureship to J I). W A receives support from the National Institute for Health Research (NIHR) Birmingham Biomedical Research Centre at the University Hospitals Birmingham NHS Foundation Trust and the University of Birmingham (Grant BRC-1215-20009). The views expressed are those of the authors and not necessarily those of the NIHR or the Department of Health and Social Care, UK. The funders of the study had no role in the: design and conduct of the study; collection, management, analysis and interpretation of the data; preparation, review or approval of the manuscript; decision to submit the manuscript for publication.

\section{Acknowledgement}

The authors thank Dr Lisa Martin, Monash University, Victoria, Australia, for kindly providing structural coordinates for their in silico model of the CYP17A1-POR-b5 complex.

\section{References}

1 Baranowski ES, Arlt W \& Idkowiak J. Monogenic disorders of adrenal steroidogenesis. Hormone Research in Paediatrics 201889 292-310. (https://doi.org/10.1159/000488034)

2 Miller WL \& Auchus RJ. The molecular biology, biochemistry, and physiology of human steroidogenesis and its disorders. Endocrine Reviews 201132 81-151. (https://doi.org/10.1210/er.2010-0013)

3 Auchus RJ, Lee TC \& Miller WL. Cytochrome b5 augments the 17,20lyase activity of human P450c17 without direct electron transfer. Journal of Biological Chemistry 1998273 3158-3165. (https://doi. org/10.1074/jbc.273.6.3158)

4 Arlt W, Walker EA, Draper N, Ivison HE, Ride JP, Hammer F, Chalder SM, Borucka-Mankiewicz M, Hauffa BP, Malunowicz EM et al. Congenital adrenal hyperplasia caused by mutant $\mathrm{P} 450$ oxidoreductase and human androgen synthesis: analytical study. Lancet 2004363 2128-2135. (https://doi.org/10.1016/S01406736(04)16503-3)

5 Flück CE, Tajima T, Pandey AV, Arlt W, Okuhara K, Verge CF, Jabs EW, Mendonca BB, Fujieda K, Miller WL et al. Mutant P450 oxidoreductase causes disordered steroidogenesis with and without Antley-Bixler syndrome. Nature Genetics 200436 228-230. (https://doi.org/10.1038/ ng1300)

6 Idkowiak J, Randell T, Dhir V, Patel P, Shackleton CHL, Taylor NF, Krone N \& Arlt W. A missense mutation in the human cytochrome b5 gene causes 46,XY disorder of sex development due to true isolated 17,20 lyase deficiency. Journal of Clinical Endocrinology and Metabolism 201297 E465-E475. (https://doi.org/10.1210/jc.2011-2413)

7 Rubtsov P, Nizhnik A, Dedov I, Kalinchenko N, Petrov V, Orekhova A, Spirin P, Prassolov V \& Tiulpakov A. Partial deficiency of $17 \alpha$-hydroxylase/17,20-lyase caused by a novel missense mutation in the canonical cytochrome heme-interacting motif. European Journal of Endocrinology 2015172 K19-K25. (https://doi.org/10.1530/EJE-140834)

8 Yanase T, Simpson ER \& Waterman MR. 17Alpha-hydroxylase/17,20-lyase deficiency: from clinical investigation to molecular definition. Endocrine Reviews 1991 12 91-108. (https://doi.org/10.1210/edrv-12-1-91)

9 Taniyama M, Tanabe M, Saito H, Ban Y, Nawata H \& Yanase T. Subtle $17 \alpha$-hydroxylase/17,20-lyase deficiency with homozygous Y201N mutation in an infertile woman. Journal of Clinical Endocrinology and Metabolism 200590 2508-2511. (https://doi.org/10.1210/jc.2004-2067)

10 Yanase T, Kagimoto M, Suzuki S, Hashiba K, Simpson ER \& Waterman MR. Deletion of a phenylalanine in the N-terminal region of human cytochrome P-450(17 alpha) results in partial combined 17 alpha-hydroxylase/17,20-lyase deficiency. Journal of Biological
Chemistry 1989264 18076-18082. (https://doi.org/10.1016/S00219258(19)84680-X)

11 Yao F, Huang S, Kang X, Zhang W, Wang P \& Tian Q. CYP17A1 mutations identified in 17 Chinese patients with $17 \alpha$-hydroxylase/17,20-lyase deficiency. Gynecological Endocrinology 201329 10-15. (https://doi.org/10.3109/09513590.2012.705373)

12 Sherbet DP, Tiosano D, Kwist KM, Hochberg Z \& Auchus RJ. CYP17 mutation E305G causes isolated 17,20-lyase deficiency by selectively altering substrate binding. Journal of Biological Chemistry 2003278 48563-48569. (https://doi.org/10.1074/jbc.M307586200)

13 Van Den Akker EL, Koper JW, Boehmer AL, Themmen AP, VerhoefPost M, Timmerman MA, Otten BJ, Drop SL \& De Jong FH. Differential inhibition of 17alpha-hydroxylase and 17,20-lyase activities by three novel missense CYP17 mutations identified in patients with P450c17 deficiency. Journal of Clinical Endocrinology and Metabolism 200287 5714-5721. (https://doi.org/10.1210/jc.2001011880)

14 Geller DH, Auchus RJ, Mendonca BB \& Miller WL. The genetic and functional basis of isolated 17,20-lyase deficiency. Nature Genetics 1997 17 201-205. (https://doi.org/10.1038/ng1097-201)

15 Shackleton C, Marcos J, Malunowicz EM, Szarras-Czapnik M, Jira P Taylor NF, Murphy N, Crushell E, Gottschalk M, Hauffa B et al. Biochemical diagnosis of Antley-Bixler syndrome by steroid analysis. American Journal of Medical Genetics: Part A 2004 128A 223-231. (https://doi.org/10.1002/ajmg.a.30104)

16 Dhir V, Reisch N, Bleicken CM, Lebl J, Kamrath C, Schwarz HP, Grötzinger J, Sippell WG, Riepe FG, Arlt W et al. Steroid $17 \alpha$-hydroxylase deficiency: functional characterization of four mutations (A174E, V178D, R440C, L465P) in the CYP17A1 gene. Journal of Clinical Endocrinology and Metabolism 200994 3058-3064. (https://doi.org/10.1210/jc.2009-0172)

17 Ioannidis NM, Rothstein JH, Pejaver V, Middha S, McDonnell SK, Baheti S, Musolf A, Li Q, Holzinger E, Karyadi D et al. REVEL: an ensemble method for predicting the pathogenicity of rare missense variants. American Journal of Human Genetics 201699 877-885. (https:// doi.org/10.1016/j.ajhg.2016.08.016)

18 Parajes S, Kamrath C, Rose IT, Taylor AE, Mooij CF, Dhir V, Grötzinger J, Arlt W \& Krone N. A novel entity of clinically isolated adrenal insufficiency caused by a partially inactivating mutation of the gene encoding for P450 side chain cleavage enzyme (CYP11A1). Journal of Clinical Endocrinology and Metabolism 201196 E1798-E1806. (https:// doi.org/10.1210/jc.2011-1277)

19 Küçükemre-Aydın B, Öğrendil-Yanar Ö, Bilge I, Baş F, Poyrazoğlu Ş, Yllmaz A, Emre S, Bundak R, Saka N \& Darendeliler F. An easily missed diagnosis: 17-alpha-hydroxylase/17,20-lyase deficiency. Turkish Journal of Pediatrics 201557 277-281.

20 Kardelen AD, Toksoy G, Baş F, Abalı ZY, Gençay G, Poyrazoğlu Ş, Bundak R, Altunoğlu U, Avcı Ş, Najaflı A et al. A rare cause of congenital adrenal hyperplasia: clinical and genetic findings and follow-up characteristics of six patients with 17-hydroxylase deficiency and two novel mutations. Journal of Clinical Research in Pediatric Endocrinology 201810 206-215. (https://doi.org/10.4274/jcrpe.0032)

21 Caulfield MP, Lynn T, Gottschalk ME, Jones KL, Taylor NF, Malunowicz EM, Shackleton CHL, Reitz RE \& Fisher DA. The diagnosis of congenital adrenal hyperplasia in the newborn by gas chromatography/mass spectrometry analysis of random urine specimens. Journal of Clinical Endocrinology and Metabolism 200287 3682-3690. (https://doi.org/10.1210/jcem.87.8.8712)

22 Holien JK, Parker MW, Conley AJ, Corbin CJ, Rodgers RJ \& Martin LL. A homodimer model can resolve the conundrum as to how cytochrome P450 oxidoreductase and cytochrome b5 compete for the same binding site on cytochrome P450c17. Current Protein and Peptide Science 201718 515-521. (https://doi.org/10.2174/138920371766616122 0142957) 
23 Burns AD, Taylor NF, Taylor DR, Bhake RC \& Rahman F. A curious case of primary amenorrhea. Clinical Chemistry 202066 1150-1154. (https://doi.org/10.1093/clinchem/hvaa083)

24 Auchus RJ. Steroid 17-hydroxylase and 17,20-lyase deficiencies, genetic and pharmacologic. Journal of Steroid Biochemistry and Molecular Biology 2017165 71-78. (https://doi.org/10.1016/j.jsbmb.2016.02.002)

25 Camats N, Üstyol A, Atabek ME, Dick B \& Flück CE. A novel CYP17A1 deletion causes a functional knockout of the steroid enzyme 17-hydroxylase and 17,20-lyase in a Turkish family and illustrates the precise role of the CYP17A1 gene. Clinical Case Reports 20153 793-797. (https://doi.org/10.1002/ccr3.343)

26 Biglieri EG, Herron MA \& Brust N. 17-Hydroxylation deficiency in man. Journal of Clinical Investigation 196645 1946-1954. (https://doi. org/10.1172/JCI105499)

27 Kurnaz E, Baykan EK, Türkyılmaz A, Yaralı O, Abalı ZY, Turan S, Bereket A, Çayır A \& Guran T. Genotypic sex and severity of the disease determine the time of clinical presentation in steroid $17 \alpha$-hydroxylase/17,20-lyase deficiency. Hormone Research in Paediatrics 202193 558-566. (https://doi.org/10.1159/000515079)

28 Müssig K, Kaltenbach S, Machicao F, Maser-Gluth C, Hartmann MF, Wudy SA, Schnauder G, Häring HU, Seif FJ \& Gallwitz B.

$17 \alpha$-Hydroxylase/17,20-lyase deficiency caused by a novel homozygous mutation (Y27Stop) in the cytochrome CYP17 gene. Journal of Clinical Endocrinology and Metabolism 200590 4362-4365. (https://doi. org/10.1210/jc.2005-0136)

29 Giraldi FP, Einaudi S, Sesta A, Verna F, Messina M, Manieri C, Menegatti E \& Ghizzoni L. POR polymorphisms are associated with 21 hydroxylase deficiency. Journal of Endocrinological Investigation 2021 1-8. (https://doi.org/10.1007/s40618-021-01527-2)

30 Kater CE \& Biglieri EG. Disorders of steroid 17 alpha-hydroxylase deficiency. Endocrinology and Metabolism Clinics of North America 1994 23 341-357. (https://doi.org/10.1016/S0889-8529(18)30101-4)

31 Hershkovitz E, Parvari R, Wudy SA, Hartmann MF, Gomes LG, Loewental N \& Miller WL. Homozygous mutation G539R in the gene for P450 oxidoreductase in a family previously diagnosed as having 17,20-lyase deficiency. Journal of Clinical Endocrinology and Metabolism 200893 3584-3588. (https://doi.org/10.1210/jc.2008-0051)

32 Kok RC, Timmerman MA, Wolffenbuttel KP, Drop SLS \& Jong FH de. Isolated 17,20-lyase deficiency due to the cytochrome b5 mutation W27X. Journal of Clinical Endocrinology and Metabolism 201095 994-999. (https://doi.org/10.1210/jc.2008-1745)

33 Leung MT, Cheung HN, Iu YP, Cheung HC, Cheung ST \& Shek CC. Isolated 17,20-lyase deficiency in a CYB5A mutated female with normal sexual development and fertility. Journal of the Endocrine Society 20194 bvz016. (https://doi.org/10.1210/jendso/bvz016)

34 Reisch N, Taylor AE, Nogueira EF, Asby DJ, Dhir V, Berry A, Krone N, Auchus RJ, Shackleton CHL, Hanley NA et al. Alternative pathway androgen biosynthesis and human fetal female virilization. PNAS 2019116 22294-22299. (https://doi.org/10.1073/ pnas.1906623116)

35 Hughes LA, Bounford KM, Webb E, Dasani P, Clokie S, Chandran H, McCarthy L, Mohamed Z, Kirk JMW, Krone N et al. Next generation sequencing (NGS) to improve the diagnosis and management of patients with disorders of sex development (DSD). Endocrine Connections 20191 100-110. (https://doi.org/10.1530/EC-18-0376)

36 Neres MS, Auchus RJ, Shackleton CHL \& Kater CE. Distinctive profile of the 17-hydroxylase and 17,20-lyase activities revealed by urinary steroid metabolomes of patients with CYP17 deficiency. Arquivos Brasileiros de Endocrinologia e Metabologia 201054 826-832. (https:// doi.org/10.1590/s0004-27302010000900009)

37 Tiosano D, Knopf C, Koren I, Levanon N, Hartmann MF, Hochberg Z $\&$ Wudy SA. Metabolic evidence for impaired 17alpha-hydroxylase activity in a kindred bearing the E305G mutation for isolate 17,20lyase activity. European Journal of Endocrinology 2008158 385-392. (https://doi.org/10.1530/EJE-07-0712)

38 Storbeck KH, Schiffer L, Baranowski ES, Chortis V, Prete A, Barnard L, Gilligan LC, Taylor AE, Idkowiak J, Arlt W et al. Steroid metabolome analysis in disorders of adrenal steroid biosynthesis and metabolism. Endocrine Reviews 201940 1605-1625. (https://doi.org/10.1210/er.201800262)

Received 16 February 2021

Revised version received 18 August 2021

Accepted 15 September 2021 